USTC-ICTS-16-14

\title{
Sound waves in the compactified D0-D4 brane system
}

\author{
1 Wenhe $\mathrm{Cai}^{*}{ }^{\dagger}$ and ${ }^{2} \mathrm{Si}$-wen $\mathrm{Li}^{*}$ \\ * Department of Modern Physics, \\ University of Science and Technology of China, \\ Hefei 230026, Anhui, China \\ $\dagger$ Interdisciplinary Center for Theoretical Study, \\ University of Science and Technology of China, \\ Hefei 230026, Anhui, China
}

\begin{abstract}
As an extension to our previous work, we study the transport properties of the Witten-Sakai-Sugimoto model in the black D4-brane background with smeared D0-branes (D0-D4/D8 system). Because of the presence of the D0-branes, in the bubble configuration this model is holographically dual to 4d QCD or Yang-Mills theory with a Chern-Simons term. And the number density of the D0-branes corresponds to the coupling constant ( $\theta$ angle) of the Chern-Simons term in the dual field theory. In this paper, we accordingly focus on small number density of the D0-branes to study the sound mode in the black D0-D4 brane system since the coupling of the Chern-Simons term should be quite weak in QCD. Then we derive its $5 \mathrm{~d}$ effective theory and analytically compute the speed of sound and the sound wave attenuation in the approach of Gauge/Gravity duality. Our result shows the speed of sound and the sound wave attenuation is modified by the presence of the D0-branes. Thus they depend on the $\theta$ angle or chiral potential in this holographic description.
\end{abstract}

\footnotetext{
${ }^{1}$ Email: edlov@mail.ustc.edu.cn

${ }^{2}$ Email: cloudk@mail.ustc.edu.cn
} 


\section{Introduction}

Since the Gauge/Gravity duality or AdS/CFT correspondence was proposed, it has become a valuable tool in analyzing near-equilibrium dynamics of the strongly coupled plasma for a long time [1, 2, 3, 4, 5. Recent years, many people believe that the QCD quark-gluon plasma (QGP) has been produced in heavy ion collision experiments at RHIC [6, 7, 8, thus the potential application of Gauge/Gravity or AdS/CFT correspondence for the hydrodynamic description of QGP becomes the most important motivation of the research in this direction. On the other hand, as one famous top-down prototype of holographic QCD, the Witten-SakaiSugimoto model [9, 10, 11] introduces a supergravity description based on the geometric background generated by $N_{c}$ D4-branes compactified on a cycle. Naturally, studying the strongly coupled hydrodynamics of QCD has become one of the most interesting aspects of this holographic model [12, 13, 14.

In the Witten-Sakai-Sugimoto model, it is realizing dual [15, 16] to four-dimensional QCD-like theory in the large- $N_{c}$ limit. Specifically, the D4-branes are compactified on a cycle with appropriate boundary conditions, therefore the dual field theory is non-conformal and non-supersymmetric which couples to the "Kaluza-Klein" field in the adjoint representation. Additionally there are $N_{f}$ species of massless flavored quarks introduced by embedding $N_{f}$ pairs of probe D8/D8-branes. In the D4 solitonic solution, the flavor D8/D8-branes are connected at the IR region, which holographically corresponds to the broken chiral symmetry in the dual field theory. And the light mesons come from the world volume theory on the connected D8/ $\overline{\mathrm{D} 8}$-branes in its low-energy effective theory.

Previously, the setup of the original Witten-Sakai-Sugimoto model was parallel implanted into the D4brane background with smeared D0-branes [17] as an extension (i.e. D0-D4/D8 brane system). And this system is holographically dual to the QCD or Yang-Mills theory with a topological term (i.e. the ChernSimons term). It would be more clear if we take into account the action of the D4-branes in the presence of the smeared D0-branes,

$$
S_{D_{4}}=-\mu_{4} \operatorname{Tr} \int d^{4} x d x^{4} e^{-\phi} \sqrt{-\operatorname{det}(\mathcal{G}+\mathcal{F})}+\mu_{4} \int C_{5}+\frac{1}{2} \mu_{4} \int C_{1} \wedge \mathcal{F} \wedge \mathcal{F}
$$

where $\mu_{4}=(2 \pi)^{-4} l_{s}^{-5}, l_{s}$ is the size of the string, $\mathcal{G}$ is the induced metric and $\mathcal{F}=2 \pi \alpha^{\prime} F$ which is proportional to the gauge field strength on the D4-brane. $C_{1}, C_{5}$ is the Romand-Romand 1- and 5- form respectively and $x^{4}$ represents the periodic direction which is wrapped on the cycle. Obviously, the Yang-Mill action comes from the leading order of the first part in (1.1) (i.e. the Dirac-Born-Infield (DBI) action) if it could be expanded by small $\mathcal{F}$. In the bubble background of the Witten-Sakai-Sugimoto in the D0-D4 background, we have the solution $C_{1} \sim \theta d x^{4}$ [17, thus the last term in (1.1) could be integrated as,

$$
\int_{S_{x^{4}}} C_{1} \sim \theta, \quad \int_{S_{x^{4}} \times \mathbb{R}^{4}} C_{1} \wedge \mathcal{F} \wedge \mathcal{F} \sim \theta \int_{\mathbb{R}^{4}} \mathcal{F} \wedge \mathcal{F}
$$

Consequently, the theory on the worldvolume of the D4-branes holographically corresponds to the QCD or Yang-Mills theory with a topological term as shown in (1.2). Phenomenologically, this topological term (1.2) may lead to some observable effects such as the Chiral Magnetic Effect (CME) (or some other effect in the glueball condensation) [18, 19. Therefore, while the experimental upper bounds on $\theta$ is quite small, the $\theta$-dependence in QCD or Yang-Mills theory is very interesting. Motivated by these, we have had many previous works on this D0-D4/D8 holographic system as 20, 21, 22, (also see other people's work about the holographic $\theta$-dependence in [23, 24]). Due to the relation between $\theta$ angle and chiral potential [25], in this manuscript, we would like to extend the study to the hydrodynamics in the black D0-D4 system although the dual field theory of this system is less clear 3 .

\footnotetext{
${ }^{3}$ While the confined geometry of the original Witten-Sakai-Sugimoto model corresponds to the confinement phase, it is less
} 
The background geometry of the black D0-D4 brane system also satisfies condition of [30, 31, so the shear viscosity $\eta$ saturates the universal viscosity bound as in [32],

$$
\frac{\eta}{s}=\frac{1}{4 \pi}
$$

where $s$ is the entropy density. It shows $\eta / s$ should not be affected by the presence of the D0-branes (in other words, the $\theta$ angle). Hence we are going to take a next step toward understanding transport phenomena in four-dimensional gauge plasma, i.e. to study the sound waves in this holographic system. And many researches of the sound waves by holographic duality could be reviewed, such as in 33, 34, also in the original Witten-Sakai-Sugimoto model [12]. Therefore as a generalization and comparison to the present results in [12, it would be quite interesting to consider the influence of the $\theta$ angle or chiral potential on the sound mode by this holographic system.

In this paper, after this introduction part, we will review the geometry of the black D0-D4 system briefly in section 2. Then we derive the 5-dimensionally effective theory of this system in section 3. Because of the presence of the D0-branes, it shows there should be a vector field in the effective theory which is additional to [12. Interestingly, this vector might relate to some other observable effect4. Moreover, we find our effective theory is also similar to the resultant theory in [35, 36]. In section 4, we study the fluctuations of the relevant fields in its effective theory and discuss how to simplify the following computations for the sound mode. Then in section 5 , the speed of the sound and the sound wave attenuation are accordingly calculated in the hydrodynamic limit. We find they are affected by the presence of the D0-branes and it may be interpreted as the modification from the $\theta$ angle (1.2) in the viewpoint of the dual field theory, or in other words, the speed of the sound and the sound wave attenuation depend on the chiral potential $\left(\mu_{5}\right)$ in this holographic description. The final section is the summary and discussion of this paper.

\section{Review of D0-D4 background}

We are going to review the black D0-D4 system briefly in this section, and some results have been presented in [17, 20, 22, 21, 37, 38. In Einstein frame, the black brane solution of $N_{c}$ D4-brane with $N_{0}$ smeared D0-branes reads [17, 38,

$$
\begin{aligned}
d s^{2} & =H_{4}^{-\frac{3}{8}}\left[-H_{0}^{-\frac{7}{8}} f_{T}(U)\left(d x^{0}\right)^{2}+H_{0}^{\frac{1}{8}} \delta_{i j} d x^{i} d x^{j}+H_{0}^{\frac{1}{8}}\left(d S^{1}\right)^{2}\right]+H_{4}^{\frac{5}{8}} H_{0}^{\frac{1}{8}}\left[\frac{d U^{2}}{f_{T}(U)}+U^{2} d \Omega_{4}^{2}\right] \\
e^{-\left(\Phi-\Phi_{0}\right)} & =H_{4}^{1 / 4} / H_{0}^{3 / 4}, \quad F_{2}=\frac{1}{\sqrt{2 !}} \frac{\mathcal{A}}{U^{4}} \frac{1}{H_{0}^{2}} d U \wedge d x^{0}, \quad F_{4}=\frac{1}{\sqrt{4 !}} \mathcal{B} \epsilon_{4} .
\end{aligned}
$$

where

$$
\begin{aligned}
\mathcal{A} & =\frac{\left(2 \pi l_{s}\right)^{7} g_{s} N_{0}}{\omega_{4} V_{4}}, \quad \mathcal{B}=\frac{\left(2 \pi l_{s}\right)^{3} g_{s} N_{c}}{\omega_{4}}, \quad e^{\Phi_{0}}=g_{s}, \\
H_{4} & =1+\frac{U_{Q_{4}}^{3}}{U^{3}}, \quad H_{0}=1+\frac{U_{Q_{0}}^{3}}{U^{3}}, \quad f_{T}(U)=1-\frac{U_{\Lambda}^{3}}{U^{3}} .
\end{aligned}
$$

clear for the deconfined geometry (black brane background) in the dual field theory. It has been discussed in [26] 27] and also in our previous study [28, 29]. In this sense, as a implanted version of the original Witten-Sakai-Sugimoto model, the dual field theory of the black D0-D4/D8 system is also less clear.

${ }^{4}$ This additional vector may be related to the Chiral Vortical Separation Effect (CVSE) [14, we would like to take a future study on it in our framework. 
we have used $g_{s}, d \Omega_{4}, \epsilon_{4}, \omega_{4}=8 \pi^{2} / 3$ to represent the string coupling, the line element, the volume form and the volume of a unit $S^{4}$ respectively. $U_{\Lambda}$ represents the position of the horizon and $V_{4}$ is the volume of the D4-brane. Notice that $x^{4}$ is the periodic direction and the D0-branes have been smeared in the $x^{i}, i=1,2,3$ and $x^{4}$ directions homogeneously. Moreover, for the reader convenience, the relation between the integration parameters $\mathcal{A}, \mathcal{B}, U_{Q_{0}}$ and $U_{Q_{4}}$ is given as [17],

$$
\mathcal{A}=3 \sqrt{U_{Q_{0}}^{3}\left(U_{Q_{0}}^{3}+U_{\Lambda}^{3}\right)}, \quad \mathcal{B}=3 \sqrt{U_{Q_{4}}^{3}\left(U_{Q_{4}}^{3}+U_{\Lambda}^{3}\right)} .
$$

By taking the near horizon limit i.e. fixing $U / \alpha^{\prime}$ and $U_{\Lambda} / \alpha^{\prime}$ where $\alpha^{\prime}=l_{s}^{2}$, we have the following relations,

$$
\begin{aligned}
U_{Q_{4}}^{3} & \rightarrow \pi \alpha^{\prime 3 / 2} g_{s} N_{c}=\frac{\beta g_{Y M}^{2} N_{c} l_{s}^{2}}{4 \pi} \equiv R^{3}, \\
H_{4}\left(U_{\Lambda}\right) & \rightarrow \frac{R^{3}}{U_{\Lambda}^{3}}, \\
\beta & \rightarrow \frac{4 \pi}{3} U_{\Lambda}^{-1 / 2} R^{3 / 2} H_{0}^{1 / 2}\left(U_{\Lambda}\right) .
\end{aligned}
$$

where $\beta$ is the size of the periodic time direction. Consequently, in the near horizon limit the black brane solution (2.1) becomes

$$
\begin{aligned}
d s^{2} & =\left(\frac{U}{R}\right)^{\frac{9}{8}}\left[-H_{0}^{-\frac{7}{8}} f_{T}(U)\left(d x^{0}\right)^{2}+H_{0}^{\frac{1}{8}} \delta_{i j} d x^{i} d x^{j}+H_{0}^{\frac{1}{8}}\left(d S^{1}\right)^{2}\right]+\left(\frac{R}{U}\right)^{\frac{15}{8}} H_{0}^{\frac{1}{8}}\left[\frac{d U^{2}}{f_{T}(U)}+U^{2} d \Omega_{4}^{2}\right] \\
e^{\Phi} & =g_{s}\left(\frac{U}{R}\right)^{3 / 4} H_{0}^{3 / 4} .
\end{aligned}
$$

Then the deformed relations in the presence of D0-branes to the variables in QCD are as follows,

$$
R^{3}=\frac{\lambda l_{s}^{2}}{2 M_{K K}} ; g_{s}=\frac{\lambda}{2 \pi M_{K K} N_{c} l_{s}} ; U_{\Lambda}=\frac{2}{9} M_{K K} \lambda l_{s}^{2} H_{0}\left(U_{\Lambda}\right) .
$$

where $\lambda=g_{Y M}^{2} N_{c}$ is the 't Hooft coupling constant and $M_{K K}$ is the mass scale.

\section{Dimensional reduction to $5 \mathrm{~d}$ theory}

As we are going to study the hydrodynamics by $\mathrm{AdS}_{5} / \mathrm{CFT}_{4}$ duality and the bulk fields in the D0-D4 background are described by 10-dimensional type IIA supergravity, so in this section let us first employ the standard Kaluza-Klein reduction on $S^{1} \times S^{4}$ as [12, 33, 36], to derive the 5-dimensionally effective theory of this system. In the Einstein frame, the 10d action of type IIA supergravity is given as,

$$
S_{I I A}=\frac{1}{2 k_{0}^{2}} \int d^{10} x \sqrt{-G}\left[\mathcal{R}^{(10)}-\frac{1}{2} \nabla_{M} \Phi \nabla^{M} \Phi-e^{\Phi / 2}\left|F_{4}\right|^{2}-e^{3 \Phi / 2}\left|F_{2}\right|^{2}\right]
$$

where $\phi$ is the dilaton and $F_{4}, F_{2}$ are the Romand-Romand 4- and 2- form field strength respectively. We have used " $G$ " to represent the determinant of 10-dimensional metric $G_{M N}$, where the indexes $(M, N)$ run from 0 to 9 . And the following ansatz of the metric would be helpful for the dimensional reduction, 


$$
\begin{aligned}
d s_{(10)}^{2} & =G_{M N} d x^{M} d x^{N} \\
& =e^{-\frac{10}{3} f} g_{a b} d x^{a} d x^{b}+e^{2 f+8 w}\left(d S^{1}\right)^{2}+e^{2 f-2 w} d \Omega_{4}^{2},
\end{aligned}
$$

where $x^{a}$ represents $x^{a}=\left\{x^{\mu}, U\right\}, \mu=0,1 \ldots 3$. Furthermore, we have assumed that the $S^{1} \times S^{4}$ dependence could be trivially reduced, it means the fields $f$ and $w$ do not depend on $S^{1} \times S^{4}$. By using the ansatz (3.2), we could obtain some useful relations which are

$$
\begin{aligned}
\sqrt{-G} & =\sqrt{-g} e^{-\frac{10}{3} f} g_{4}^{1 / 2}, \\
\sqrt{-G}\left|F_{4}\right|^{2} & =\mathcal{B}^{2} e^{-8(f-w)} \sqrt{-g} e^{-\frac{10}{3} f} g_{4}^{1 / 2}, \\
\sqrt{-G}\left|F_{2}\right|^{2} & =\sqrt{-g} F_{a b} F_{c d} g^{a c} g^{b d} e^{\frac{10}{3} f} g_{4}^{1 / 2}, \\
\sqrt{-G} \nabla_{M} \Phi \nabla^{M} \Phi & =\sqrt{-g} g_{4}^{1 / 2} \nabla_{a} \Phi \nabla^{a} \Phi
\end{aligned}
$$

where $g_{4}$ represents the determinant of the metric on $S^{4}$ and $g_{a b}$ is the $5 \mathrm{~d}$ metric. The relation between $10 \mathrm{~d}$ $\mathcal{R}^{(10)}$ and $5 \mathrm{~d} \mathcal{R}^{(5)}$ curvature scalar is given a: 5

$$
\mathcal{R}^{(10)}=e^{\frac{10}{3} f}\left[\mathcal{R}^{(5)}-20 g^{a b} \partial_{a} w \partial_{b} w-\frac{40}{3} g^{a b} \partial_{a} f \partial_{b} f\right]+12 e^{-2(f-w)} .
$$

After inserting (3.4) into (3.1) and integrating over $S^{1} \times S^{4}$, we obtain the 5 d effective action which takes the following form,

$$
S_{5 d}=\frac{\pi \mathcal{V}_{4}}{k_{0}^{2}} \int d^{5} x \sqrt{-g}\left[\mathcal{R}^{(5)}-\frac{1}{2} g^{a b} \partial_{a} \Phi \partial_{b} \Phi-20 g^{a b} \partial_{a} w \partial_{b} w-\frac{40}{3} g^{a b} \partial_{a} f \partial_{b} f-\mathcal{P}-e^{\frac{10}{3} f+\frac{3}{2} \Phi} F_{a b} F_{c d} g^{a c} g^{b d}\right],
$$

where $\mathcal{V}_{4}$ represents the volume of the 4 -sphere and

$$
\mathcal{P}=\mathcal{B}^{2} e^{\frac{\Phi}{2}-\frac{34}{3} f+8 w}-12 e^{-\frac{16}{3} f+2 w} .
$$

The equations of motion for $\Phi, w, f$ and $g_{a b}$ could be obtained from which are as follows,

$$
\begin{aligned}
& g^{a b} \nabla_{a} \nabla_{b} f-\frac{3}{80} \frac{\partial \mathcal{P}}{\partial f}-\frac{1}{8} e^{\frac{10}{3} f+\frac{3}{2} \Phi} F_{a b} F_{c d} g^{a c} g^{b d}=0, \\
& g^{a b} \nabla_{a} \nabla_{b} w-\frac{1}{40} \frac{\partial \mathcal{P}}{\partial w}=0, \\
& g^{a b} \nabla_{a} \nabla_{b} \Phi-\frac{\partial \mathcal{P}}{\partial \Phi}-\frac{3}{2} e^{\frac{10}{3} f+\frac{3}{2} \Phi} F_{a b} F_{c d} g^{a c} g^{b d}=0, \\
& \partial_{a}\left[\sqrt{-g} e^{\frac{10}{3} f+\frac{3}{2} \Phi} F^{a b}\right]=0, \\
& \frac{1}{2} \partial_{a} \Phi \partial_{b} \Phi+20 \partial_{a} w \partial_{b} w+\frac{40}{3} \partial_{a} f \partial_{b} f+\frac{1}{3} g_{a b} \mathcal{P}+\left(2 F_{c a} F_{b}^{c}-\frac{1}{3} g_{a b} F_{c d} F^{c d}\right) e^{\frac{10}{3} f+\frac{3}{2} \Phi}=\mathcal{R}_{a b}^{(5 d)} .
\end{aligned}
$$

\footnotetext{
${ }^{5}$ We will not give the full relation in (3.4) since there would be some additional total derivatives if imposing the full relation of (3.4) to the action (3.1). Those terms have thus been dropped off. So only the relevant terms are given in (3.4).
} 
We have used $F_{a b}$ to represent the components of the Romand-Romand 2-form $F_{2}$. Then let us consider the $5 \mathrm{~d}$ ansatz of the metric as

$$
d s_{(5)}^{2}=-c_{1}^{2} d t^{2}+c_{2}^{2} \delta_{i j} d x^{i} d x^{j}+c_{3}^{2} d U^{2}
$$

which is obtained from the following corresponding $10 \mathrm{~d}$ metric (3.2),

$$
d s_{(10)}^{2}=e^{-\frac{10}{3} f}\left[-c_{1}^{2} d t^{2}+c_{2}^{2} \delta_{i j} d x^{i} d x^{j}+c_{3}^{2} d U^{2}\right]+e^{2 f+8 w}\left(d S^{1}\right)^{2}+e^{2(f-w)} d \Omega_{4}^{2} .
$$

Comparing (3.9) with the black brane solution of the Witten-Sakai-Sugimoto model in the D0-D4 background (2.1), it leads to the following relations,

$$
\begin{aligned}
f & =\frac{1}{16} \log H_{0}+\frac{13}{80} \log U, \\
w & =\frac{1}{10} \log U, \\
c_{1} & =f_{T}^{1 / 2} U^{5 / 6} H_{0}^{-1 / 3} \\
c_{2} & =H_{0}^{1 / 6} U^{5 / 6} \\
c_{3} & =f_{T}^{-1 / 2} H_{0}^{1 / 6} U^{-2 / 3} .
\end{aligned}
$$

where we have set $g_{s}=R=1$ for convenience (as a comparison with [12]), so that $\mathcal{B}=\sqrt{\frac{9}{2}}$. And one can verify the reduced functions in (3.10) satisfies the $5 \mathrm{~d}$ effective equations of motion (3.7) consistently. Consequently we obtain the $5 \mathrm{~d}$ effective action (3.5) and its solution (3.9) (3.10) of our D0-D4 brane system. But as a difference from the original D4-brane system, there is an additional vector field $C_{a}$ in the low energy effective theory whose field strength is the Romand-Romand 2-form defined as $F_{a b}=\partial_{a} C_{b}-\partial_{b} C_{a}$.

\section{Fluctuations}

In this section, let us study the fluctuations of the relevant fields in the black D0-D4 background by replacing6,

$$
\begin{aligned}
g_{a b} & \rightarrow g_{a b}+h_{a b}, \\
f & \rightarrow f+\delta f, \\
w & \rightarrow w+\delta w, \\
\Phi & \rightarrow \Phi+\delta \Phi, \\
C_{a} & \rightarrow C_{a}+\delta C_{a} .
\end{aligned}
$$

where $\left\{h_{a b}, \delta f, \delta w, \delta \Phi, \delta C_{a}\right\}$ are the fluctuations while $\left\{g_{a b}, f, w, \Phi, C_{a}\right\}$ are the background configurations of the D0-D4 system i.e. the classical solution of the equations of motion (3.7). For the fluctuations of the metric, we are going to choose the following gauge as [12, 36, 34, 33],

$$
h_{a U}=0 .
$$

\footnotetext{
${ }^{6}$ By the solution for the D0-D4 background (2.1), we have assumed that only one component of $C_{a}$ is nonzero which is $C_{t}$.
} 
Furthermore, we have assumed that the fluctuations of the metric depends on $\{t, z, U\} 77$ only i.e. the system we are considering is $O(2)$ rotationally symmetric in the $x-y$ plane.

In the linearized case, the following sets of the metric are decoupled from each other because of the $O(2)$ symmetry [34,

$$
\begin{array}{r}
\left\{h_{12}\right\}, \\
\left\{h_{11}-h_{22}\right\}, \\
\left\{h_{01}, h_{13}\right\}, \\
\left\{h_{02}, h_{23}\right\}, \\
\left\{h_{00}, h_{\alpha \alpha}=h_{11}+h_{22}, h_{03}, h_{33}\right\} .
\end{array}
$$

While the first three sets in (4.3) are related to the shear modes, the last set corresponds to the sound waves which is the concern in this manuscript. Besides, there are additional fluctuations as $\left\{\delta f, \delta w, \delta \Phi, \delta C_{a}\right\}$ from the dimension-reductional scalars and vector. As a comparison, let us employ the similar conventions as $12,33,36$ by introducing 8 ,

$$
\begin{gathered}
h_{00}=e^{-i \omega t+i q x_{3}} c_{1}^{2} H_{t t}, h_{03}=e^{-i \omega t+i q x_{3}} c_{2}^{2} H_{t z}, \\
h_{\alpha \alpha}=e^{-i \omega t+i q x_{3}} c_{2}^{2} H_{\alpha \alpha}, h_{33}=e^{-i \omega t+i q x_{3}} c_{2}^{2} H_{z z}, \\
\delta f=e^{-i \omega t+i q x_{3}} \mathcal{F}, \delta w=e^{-i \omega t+i q x_{3}} \mathcal{W}, \\
\delta \Phi=e^{-i \omega t+i q x_{3}} \varphi, \delta C_{a}=e^{-i \omega t+i q x_{3}} \mathcal{C}_{a} .
\end{gathered}
$$

where the definition of the functions $c_{1}$ and $c_{2}$ has been given in (3.10) and $\left\{H_{t t}, H_{t z}, H_{\alpha \alpha}, H_{z z}, \mathcal{F}, \mathcal{W}, \varphi, \mathcal{C}_{a}\right\}$ are the functions which depend on the radial coordinate $U$ only. By inserting (4.1) (4.4) into (3.7) and expanding all the equations of motion at a linearized level, we obtain five ordinary differential equations as in the Appendix. And the relevant equations are collected as follows once we evaluate (A1) - (A5) by (3.9) (3.10) and (4.4), which are

$$
\begin{aligned}
0= & H_{t t}^{\prime \prime}+\left[\ln \frac{c_{1}^{2} c_{2}^{3}}{c_{3}}\right]^{\prime} H_{t t}^{\prime}-\left[\ln c_{1}\right]^{\prime} H_{i i}^{\prime}-\frac{c_{3}^{2}}{c_{1}^{2}}\left(q^{2} \frac{c_{1}^{2}}{c_{2}^{2}} H_{t t}+\omega^{2} H_{i i}+2 \omega q H_{t z}\right) \\
& -\frac{2}{3} c_{3}^{2}\left(\frac{\partial \mathcal{P}}{\partial f} \mathcal{F}+\frac{\partial \mathcal{P}}{\partial w} \mathcal{W}+\frac{\partial \mathcal{P}}{\partial \Phi} \varphi\right)+\left(\frac{6 c_{1}^{\prime} c_{2}^{\prime}}{c_{1} c_{2}}-\frac{2 c_{1}^{\prime} c_{3}^{\prime}}{c_{1} c_{3}}+\frac{2 c_{1}^{\prime \prime}}{c_{1}}+\frac{2}{3} c_{3}^{2} \mathcal{P}\right) H_{t t} \\
& +\frac{4 F_{t U}}{9 c_{1}^{2}} e^{\frac{10}{3} f+\frac{3}{2} \Phi}\left(12 \hat{F}_{t U}+20 F_{t U} \mathcal{F}+9 F_{t U} \varphi\right), \\
0= & H_{t z}^{\prime \prime}+\left[\ln \frac{c_{2}^{5}}{c_{1} c_{3}}\right]^{\prime} H_{t z}^{\prime}+q \omega \frac{c_{3}^{2}}{c_{2}^{2}} H_{\alpha \alpha}+\left(\frac{2 c_{1}^{\prime} c_{2}^{\prime}}{c_{1} c_{2}}+\frac{4 c_{2}^{\prime 2}}{c_{2}^{2}}-\frac{2 c_{2}^{\prime} c_{3}^{\prime}}{c_{2} c_{3}}+\frac{2 c_{2}^{\prime \prime}}{c_{2}^{2}}+\frac{2}{3} c_{3}^{2} \mathcal{P}\right) H_{t z} \\
& \frac{4 F_{t U}}{3 c_{1}^{2}} e^{\frac{10}{3} f+\frac{3}{2} \Phi}\left(3 \hat{F}_{3 U} \frac{c_{1}^{2}}{c_{2}^{2}}+F_{t U} H_{t z}\right),
\end{aligned}
$$

\footnotetext{
${ }^{7}$ The coordinate $x^{\mu}$ could be identified as $\{t, x, y, z\}$.

${ }^{8}$ It would not be confused with (1.1) (1.2) if we use the same $\mathcal{F}$ to represent the fluctuation of the function $f$ here.
} 


$$
\begin{aligned}
& 0=H_{a a}^{\prime \prime}+\left[\ln \frac{c_{1} c_{2}^{5}}{c_{3}}\right]^{\prime} H_{a a}^{\prime}+\frac{c_{3}^{2}}{c_{1}^{2}}\left(\omega^{2}-q^{2} \frac{c_{1}^{2}}{c_{2}^{2}}\right) H_{a a}+\left(H_{z z}^{\prime}-H_{t t}^{\prime}\right)\left[\ln c_{2}^{2}\right]^{\prime} \\
& +\left(\frac{4 c_{1} c_{3} c_{2}^{\prime 2}+2 c_{2} c_{3} c_{1}^{\prime} c_{2}^{\prime}-2 c_{2} c_{1} c_{2}^{\prime} c_{3}^{\prime}}{c_{1} c_{2}^{2} c_{3}}+\frac{2 c_{2}^{\prime \prime}}{c_{2}}+\frac{2}{3} c_{3}^{2} \mathcal{P}\right) H_{a a}+\frac{4}{3} c_{3}^{2}\left(\frac{\partial \mathcal{P}}{\partial f} \mathcal{F}+\frac{\partial \mathcal{P}}{\partial w} \mathcal{W}+\frac{\partial \mathcal{P}}{\partial \Phi} \varphi\right) \\
& +\frac{4 F_{t U}}{9 c_{1}^{2}} e^{\frac{10}{3} f+\frac{3}{2} \Phi}\left(20 F_{t U} \mathcal{F}+12 \hat{F}_{t U}+6 F_{t U} H_{t t}+3 F_{t U} H_{a a}+9 F_{t U} \varphi\right), \\
& 0=H_{z z}^{\prime \prime}+\left[\ln \frac{c_{1} c_{2}^{4}}{c_{3}}\right]^{\prime} H_{z z}^{\prime}+\left(H_{a a}^{\prime}-H_{t t}^{\prime}\right)\left[\ln c_{2}\right]^{\prime}+\frac{c_{3}^{2}}{c_{1}^{2}}\left[\omega^{2} H_{z z}+2 \omega q H_{t z}+q^{2} \frac{c_{1}^{2}}{c_{2}^{2}}\left(H_{t t}-H_{a a}\right)\right] \\
& +H_{z z}\left(\frac{c_{1}^{\prime} c_{2}^{\prime}}{c_{1} c_{2}}+\frac{c_{2}^{\prime 2}}{c_{2}^{2}}-\frac{c_{2}^{\prime} c_{3}^{\prime}}{c_{2} c_{3}}+\frac{2 c_{2}^{\prime \prime}}{c_{2}}+\frac{2}{3} c_{3}^{2} \mathcal{P}\right)+\frac{2}{3} c_{3}^{2}\left(\frac{\partial \mathcal{P}}{\partial f} \mathcal{F}+\frac{\partial \mathcal{P}}{\partial w} \mathcal{W}+\frac{\partial \mathcal{P}}{\partial \Phi} \varphi\right) \\
& +\frac{2 F_{t U}}{9 c_{1}^{2}} e^{\frac{10}{3} f+\frac{3}{2} \Phi}\left(12 \hat{F}_{t U}+20 F_{t U} \mathcal{F}+6 F_{t U} H_{t t}+6 F_{t U} H_{z z}+9 F_{t U} \varphi\right), \\
& 0=\mathcal{F}^{\prime \prime}+\left[\ln \frac{c_{1} c_{2}^{3}}{c_{3}}\right]^{\prime} \mathcal{F}^{\prime}+\frac{1}{2} f^{\prime}\left(H_{i i}^{\prime}-H_{t t}^{\prime}\right)+\frac{c_{3}^{2}}{c_{1}^{2}}\left(\omega^{2}-q^{2} \frac{c_{1}^{2}}{c_{2}^{2}}\right) \mathcal{F}-\frac{3}{80} c_{3}^{2}\left(\frac{\partial^{2} \mathcal{P}}{\partial f^{2}} \mathcal{F}+\frac{\partial^{2} \mathcal{P}}{\partial f \partial w} \mathcal{W}+\frac{\partial^{2} \mathcal{P}}{\partial f \partial \Phi} \varphi\right) \\
& +\frac{F_{t U}}{24 c_{1}^{2}} e^{\frac{10}{3} f+\frac{3}{2} \Phi}\left(12 \hat{F}_{t U}+20 F_{t U} \mathcal{F}+6 F_{t U} H_{t t}+9 F_{t U} \varphi\right) \\
& 0=\mathcal{W}^{\prime \prime}+\left[\ln \frac{c_{1} c_{2}^{3}}{c_{3}}\right]^{\prime} \mathcal{W}^{\prime}+\frac{1}{2} w^{\prime}\left(H_{i i}^{\prime}-H_{t t}^{\prime}\right)+\frac{c_{3}^{2}}{c_{1}^{2}}\left(\omega^{2}-q^{2} \frac{c_{1}^{2}}{c_{2}^{2}}\right) \mathcal{W} \\
& -\frac{1}{40} c_{3}^{2}\left(\frac{\partial^{2} \mathcal{P}}{\partial w \partial f} \mathcal{F}+\frac{\partial^{2} \mathcal{P}}{\partial w^{2}} \mathcal{W}+\frac{\partial^{2} \mathcal{P}}{\partial w \partial \Phi} \varphi\right) \\
& 0=\varphi^{\prime \prime}+\left[\ln \frac{c_{1} c_{2}^{3}}{c_{3}}\right]^{\prime} \varphi^{\prime}+\frac{1}{2} \Phi^{\prime}\left(H_{i i}^{\prime}-H_{t t}^{\prime}\right)+\frac{c_{3}^{2}}{c_{1}^{2}}\left(\omega^{2}-q^{2} \frac{c_{1}^{2}}{c_{2}^{2}}\right) \varphi-c_{3}^{2}\left(\frac{\partial^{2} \mathcal{P}}{\partial \Phi \partial f} \mathcal{F}+\frac{\partial^{2} \mathcal{P}}{\partial \Phi \partial w} \mathcal{W}+\frac{\partial^{2} \mathcal{P}}{\partial \Phi^{2}} \varphi\right) \\
& +\frac{F_{t U}}{2 c_{1}^{2}} e^{\frac{10}{3} f+\frac{3}{2} \Phi}\left(12 \hat{F}_{t U}+20 F_{t U} \mathcal{F}+6 F_{t U} H_{t t}+9 F_{t U} \varphi\right)
\end{aligned}
$$

where $\hat{F}_{a b}=\mathcal{A} e^{i \omega t-i q x_{3}}\left(\partial_{a} \delta C_{b}-\partial_{b} \delta C_{a}\right) \sqrt[9]{9}$. Besides, there are three additional first order constraints which comes by associating with the (partially) fixed diffeomorphism invarianct10,

\footnotetext{
${ }^{9} \mathrm{By}$ this definition, there would be an " $i$ " factor in $\hat{F}_{t z}$ once we calculate the derivative respected to $z$ or $x_{3}$. In this sense, (4.13) is a real equation although there is an " $i$ " factor.

${ }^{10}$ In fact there are more additional equations from the vanished components of linearized Ricci tensor. We have checked that those equations determine the vanished components of $\delta F_{a b}$. As a result, the nonzero and relevant components of $\delta F_{a b}$ mixed to the sound mode are only $\delta F_{t z}, \delta F_{z U}, \delta F_{t U}$. So the nonzero components of $\delta C_{a}$ could be $\delta C_{t}$ only if $C_{U}, \delta C_{U}$ are gauged by $C_{U}, \delta C_{U}=0$.
} 


$$
\begin{gathered}
0=\omega\left(H_{i i}^{\prime}+\left[\ln \frac{c_{2}}{c_{1}}\right]^{\prime} H_{i i}\right)+q\left(H_{t z}^{\prime}+2\left[\ln \frac{c_{2}}{c_{1}}\right]^{\prime} H_{t z}\right) \\
+\omega\left(\frac{80}{3} f^{\prime} \mathcal{F}+40 w^{\prime} \mathcal{W}+\Phi^{\prime} \varphi\right), \\
0=q\left(H_{t t}^{\prime}-\left[\ln \frac{c_{2}}{c_{1}}\right]^{\prime} H_{t t}\right)+\frac{c_{2}^{2}}{c_{1}^{2}} \omega H_{t z}^{\prime}-q H_{a a}^{\prime}+4 i \hat{F}_{t z} \frac{F_{t U}}{c_{1}^{2}} e^{\frac{10}{3} f+\frac{3}{2} \Phi} \\
-q\left(\frac{80}{3} f^{\prime} \mathcal{F}+40 w^{\prime} \mathcal{W}+\Phi^{\prime} \varphi\right), \\
=\left[\ln c_{1} c_{2}^{2}\right]^{\prime} H_{i i}^{\prime}-\left[\ln c_{2}^{3}\right]^{\prime} H_{t t}^{\prime}+\frac{c_{3}^{2}}{c_{1}^{2}}\left[\omega^{2} H_{i i}+2 \omega q H_{t z}+q^{2} \frac{c_{1}^{2}}{c_{2}^{2}}\left(H_{t t}-H_{a a}\right)\right] \\
+c_{3}^{2}\left(\frac{\partial \mathcal{P}}{\partial f} \mathcal{F}+\frac{\partial \mathcal{P}}{\partial w} \mathcal{W}+\frac{\partial \mathcal{P}}{\partial \Phi} \varphi\right)-\left(\frac{80}{3} f^{\prime} \mathcal{F}^{\prime}+40 w^{\prime} \mathcal{W}^{\prime}+\Phi^{\prime} \varphi^{\prime}\right)+\frac{2}{3} c_{3}^{2} \mathcal{P}\left(H_{i i}-H_{t t}\right) \\
+\left(\frac{c_{1}^{\prime} c_{2}^{\prime}}{c_{1} c_{2}}+\frac{2 c_{2}^{\prime 2}}{c_{2}^{2}}-\frac{c_{2}^{\prime} c_{3}^{\prime}}{c_{2} c_{3}}+\frac{c_{2}^{\prime \prime}}{c_{2}}\right) H_{i i}+\left(\frac{c_{1}^{\prime} c_{3}^{\prime}}{c_{1} c_{3}}-\frac{3 c_{1}^{\prime} c_{2}^{\prime}}{c_{1} c_{2}}-\frac{c_{1}^{\prime \prime}}{c_{1}}\right) H_{t t} \\
+\frac{F_{t U}}{3 c_{1}^{2}} e^{\frac{10}{3} f+\frac{3}{2} \Phi}\left(12 \hat{F}_{t U}+20 F_{t U} \mathcal{F}+10 F_{t U} H_{t t}+2 F_{t U} H_{a a}+2 F_{t U} H_{z z}+9 F_{t U} \varphi\right) .
\end{gathered}
$$

Notice that, we do not give the relations of the fluctuations from the equation of motion for the vector field $C_{a}$, since this vector part corresponds to diffusive or transverse channel 34, which are less relevant to the sound modes [34. Therefore we will not attempt to discuss more about the vector part thus we can simply set $\delta C_{a}=0$ if studying the sound mode only. Nevertheless, the surviving equations of motion from (4.5) - (4.14) are still complicated. On the other hand, since the parameter $\mathcal{A}$ is related the coupling constant of the topological term in the dual field theory as (1.2), which is actually very small, thus it simplifies the calculation greatly if we only consider the leading order in small $\mathcal{A}$ expansion of all the equations in (4.5) (4.14). Then if we introduce the gauge invariant variables as [12, 33, 34, 36,

$$
\begin{aligned}
Z_{H} & =4 \frac{q}{\omega} H_{t z}+2 H_{z z}-H_{a a}\left(1-\frac{q^{2}}{\omega^{2}} \frac{c_{1}^{\prime} c_{1}}{c_{2}^{\prime} c_{2}}\right)+2 \frac{q^{2}}{\omega^{2}} \frac{c_{1}^{2}}{c_{2}^{2}} H_{t t}, \\
Z_{f} & =\mathcal{F}-\frac{f^{\prime}}{\left[\ln c_{2}^{4}\right]^{\prime}} H_{a a}, \\
Z_{w} & =\mathcal{W}-\frac{w^{\prime}}{\left[\ln c_{2}^{4}\right]^{\prime}} H_{a a}, \\
Z_{\Phi} & =\varphi-\frac{\Phi^{\prime}}{\left[\ln c_{2}^{4}\right]^{\prime}} H_{a a} .
\end{aligned}
$$

with a new coordinate

$$
x=\frac{c_{1}}{c_{2}}
$$


then we find the decoupled equations of motion for $Z$ ' s by imposing (4.5) - (4.14) in small $\mathcal{A}$ expansion as

$$
\begin{aligned}
0 & =\frac{d^{2} Z_{H}}{d x^{2}}+\left[\frac{3 q^{2}\left(2 x^{2}-1\right)+5 \omega^{2}}{x\left(5 \omega^{2}-q^{2}\left(3+2 x^{2}\right)\right)}+\mathcal{A}^{2} h_{1}(x)\right] \frac{d Z_{H}}{d x} \\
& +\left[\frac{4}{9} \frac{\left(-\omega^{2}+q^{2} x^{2}\right)\left(q^{2}\left(3+2 x^{2}\right)-5 \omega^{2}\right)-18 q^{2} U_{\Lambda} x^{2}\left(1-x^{2}\right)^{5 / 3}}{\left(5 \omega^{2}-q^{2}\left(3+2 x^{2}\right)\right)\left(1-x^{2}\right)^{5 / 3} x^{2} U_{\Lambda}}+\mathcal{A}^{2} h_{2}(x)\right] Z_{H} \\
& +\left[\frac{4}{15} \frac{q^{2}\left(-3 q^{2}+5 \omega^{2}\right)}{\omega^{2}\left(5 \omega^{2}-q^{2}\left(3+2 x^{2}\right)\right)}+\mathcal{A}^{2} g_{1}(x)\right] \kappa+\mathcal{O}\left(\mathcal{A}^{4}\right), \\
0 & =\frac{d^{2} \kappa}{d x^{2}}+\left[\frac{1}{x}+\mathcal{A}^{2} g_{2}(x)\right] \frac{d \kappa}{d x}+\left[\frac{4\left(\omega^{2}-q^{2} x^{2}\right)}{9 U_{\Lambda} x^{2}\left(1-x^{2}\right)^{5 / 3}}+\mathcal{A}^{2} g_{3}(x)\right] \kappa+\mathcal{O}\left(\mathcal{A}^{4}\right) .
\end{aligned}
$$

where

$$
\begin{aligned}
\kappa= & 48 Z_{w}+9 Z_{\phi}+52 Z_{f}, \\
h_{1}(x)= & \frac{2\left(q^{4}\left(54-33 x^{2}+84 x^{4}+20 x^{6}\right)-10 q^{2}\left(9+16 x^{4}\right) \omega^{2}+125 x^{2} \omega^{4}\right)}{45 x U_{\Lambda}^{6}\left(q^{2}\left(3+2 x^{2}\right)-5 \omega^{2}\right)^{2}}, \\
h_{2}(x)= & \frac{4}{1215 x^{2}\left(-1+x^{2}\right)^{2} U_{\Lambda}^{7}\left(q^{2}\left(3+2 x^{2}\right)-5 \omega^{2}\right)} \times \\
& {\left[5 q^{6} x^{2}\left(1-x^{2}\right)^{1 / 3}\left(-27+36 x^{4}+16 x^{6}\right)+125\left(3-4 x^{2}\right)\left(1-x^{2}\right)^{1 / 3} \omega^{6}\right.} \\
& +15 q^{2} \omega^{2}\left(27\left(-1+x^{2}\right)^{3}\left(-3+2 x^{2}\right) U_{\Lambda}+5\left(1-x^{2}\right)^{1 / 3}\left(-6-x^{2}+12 x^{4}\right) \omega^{2}\right) \\
& \left.-3 q^{4}\left(27\left(-1+x^{2}\right)^{3}\left(-9+4 x^{2}\right) U_{\Lambda}+5\left(1-x^{2}\right)^{1 / 3}\left(-9-30 x^{2}+32 x^{4}+32 x^{6}\right) \omega^{2}\right)\right] .
\end{aligned}
$$

We will not give the explicit formula of the functions $g_{1,2,3}(x)$ used in (4.17) since they are too messy and lengthy. Moreover, in the next section, it would be clear that the functions $g_{1,2,3}(x)$ are actually less useful to the calculations for the sound mode, because the sound mode are relevant to $Z_{H}$ only.

\section{$5 \quad$ Hydrodynamic limit in small $\mathcal{A}$ expansion}

In this section, let us study the physical fluctuation equations (4.17) in the hydrodynamic limit i.e. $\omega \rightarrow$ $0, q \rightarrow 0$ but $\frac{\omega}{q}$ is fixed as a constant. As many discussions, only the leading and next-to-leading solution (in small $q$ expansion) of (4.17) is needed. On the hand, since the sound mode are relevant to $Z_{H}$ instead of $\kappa$, so similar to the discussions and calculations in [12, 33, 34, 36, we can simply choose $\kappa=0$ as the solution for (4.17) consistently 11. And for $Z_{H}$ we find that at the horizon $x \rightarrow 0_{+}, Z_{H} \rightarrow x^{ \pm \frac{i \omega}{2 \pi T}} x^{-\mathcal{A}}$, 12 . By imposing the incoming boundary condition on all physical modes, we assume that

\footnotetext{
${ }^{11}$ It has been discussed that $\kappa=0$ could be a solution for $\mathcal{A}=0$ case [12, so it is also consistent with this solution for $\kappa$ in small $\mathcal{A}$ case

${ }^{12}$ As $\mathcal{A}>0$, it means $x^{-\mathcal{A}}$ is also singular if $x \rightarrow 0$. This behavior at the horizon is a bit different from the original D4-brane system, however it should be consistent in the small $\mathcal{A}$ limit.
} 


$$
Z_{H}=x^{-\frac{i \omega}{2 \pi T}} x^{-\mathcal{A}} z_{H}
$$

where $z_{H}$ must be regular at horizon. Additionally, since we are interested in the hydrodynamic pole dispersion-relation in the stress-energy correlation, it would be convenient to parameterize the $\boldsymbol{\omega}$ and $\mathfrak{q}$ as

$$
\boldsymbol{\omega}=v_{s} \mathfrak{q}-i \mathfrak{q}^{2} \Gamma
$$

where

$$
\boldsymbol{\omega}=\frac{\omega}{2 \pi T}, \quad \mathfrak{q}=\frac{q}{2 \pi T},
$$

and $v_{s}, \Gamma$ is the speed of sound, the sound wave attenuation respectively, which would be determined from the pole dispersion relation. Without the loss of generality, we can choose the boundary condition for $z_{H}$ as [12,

$$
\left.z_{H}\right|_{x \rightarrow 0_{+}}=1,\left.z_{H}\right|_{x \rightarrow 1_{-}}=0 .
$$

By expanding $z_{H}$ with small $\mathfrak{q}$, we assume

$$
z_{H}=z_{H, 0}+i \mathfrak{q} z_{H, 1} .
$$

Inserting (5.1) - (5.4) into (4.17) in small $\mathfrak{q}$ and $\mathcal{A}$ expansion with $\kappa=0$, we obtain the following equations for $z_{H, 0}$ and $z_{H, 1}$ as

$$
\begin{aligned}
0= & z_{H, 0}^{\prime \prime}-\frac{6 x^{2}+5 v_{s}^{2}-3}{x\left(2 x^{2}-5 v_{s}^{2}+3\right)} z_{0, H}^{\prime}+\frac{8}{2 x^{2}-5 v_{s}^{2}+3} z_{0, H} \\
& +\mathcal{A}\left[\left(\frac{1}{x^{2}}+\frac{6 x^{2}+5 v_{s}^{2}-3}{x^{2}\left(2 x^{2}-5 v_{s}^{2}+3\right)}\right) z_{0, H}-\frac{2}{x} z_{0, H}^{\prime}\right]
\end{aligned}
$$

for leading order in $\mathcal{O}\left(\mathfrak{q}^{0}\right)$ and

$$
\begin{aligned}
0= & z_{H, 1}^{\prime \prime}+\frac{3-5 v_{s}^{2}-6 x^{2}}{x\left(3-5 v_{s}^{2}+2 x^{2}\right)} z_{H, 1}^{\prime}+\frac{8}{3-5 v_{s}^{2}+2 x^{2}} z_{H, 1} \\
& +\frac{2 v_{s}\left(40 x^{2} \Gamma+20 x^{2} v_{s}^{2}-25 v_{s}^{4}+30 v_{s}^{2}-4 x^{4}-12 x^{2}-9\right)}{x\left(2 x^{2}-5 v_{s}^{2}+3\right)^{2}} z_{H, 0}^{\prime} \\
& -\frac{8 v_{s}\left(-2 x^{2}+5 v_{s}^{2}-3+10 \Gamma\right)}{\left(2 x^{2}-5 v_{s}^{2}+3\right)^{2}} z_{H, 0}-\mathcal{A}\left[\frac{2 z_{H, 1}^{\prime}}{x}-\frac{8}{3-5 v_{s}^{2}+2 x^{2}} z_{H, 1}\right. \\
& \left.+\frac{2 v_{s}\left(40 x^{2} \Gamma+20 x^{2} v_{s}^{2}-25 v_{s}^{4}+30 v_{s}^{2}-4 x^{4}-12 x^{2}-9\right)}{x^{2}\left(2 x^{2}-5 v_{s}^{2}+3\right)^{2}} z_{H, 0}\right] .
\end{aligned}
$$

for next to leading order in $\mathcal{O}\left(\mathfrak{q}^{1}\right)$. Then the solution for equation (5.6) can be obtained as, 


$$
\begin{aligned}
z_{H, 0}= & \frac{\left(3-3 \mathcal{A}-5 v_{s}^{2}+5 \mathcal{A} v_{s}^{2}-2 x^{2}-2 \mathcal{A} x^{2}\right) C_{1}}{1-5 \mathcal{A}-5 v_{s}^{2}+5 \mathcal{A} v_{s}^{2}}+\frac{\left(3-3 \mathcal{A}-5 v_{s}^{2}+5 \mathcal{A} v_{s}^{2}-2 x^{2}-2 \mathcal{A} x^{2}\right)}{2(1+\mathcal{A})^{2}\left(1-5 \mathcal{A}-5 v_{s}^{2}+5 \mathcal{A} v_{s}^{2}\right)} \\
& \times x^{\mathcal{A}}\left[\frac{1}{\mathcal{A}}-\frac{4\left(-3+5 v_{s}^{2}\right)}{(-1+\mathcal{A})\left(-3+5 v_{s}^{2}+2 x^{2}+\mathcal{A}\left(3-5 v_{s}^{2}+2 x^{2}\right)\right)}\right] C_{2} .
\end{aligned}
$$

where $C_{1,2}$ are two integration constants. We impose the boundary condition (5.4) for $z_{H, 0}$,

$$
\left.z_{H, 0}\right|_{x \rightarrow 1^{-}}=0,
$$

Besides, in order to compare our solution (5.8) with [12, we further require

$$
\left.z_{H, 0}\right|_{x \rightarrow 0^{-}}=1 \text {. }
$$

Thus the relation between the integration constants $C_{1,2}$ is obtained as,

$$
C_{2}=-\frac{2 \mathcal{A}\left(-1+\mathcal{A}^{2}\right)^{2} C_{1}^{2}}{4 \mathcal{A}+C_{1}-2 \mathcal{A} C_{1}+\mathcal{A}^{2} C_{1}}
$$

The solution (5.8) should be definitely able to return to [12] in small $\mathcal{A}$ limit, in this sense we have the following extra relations

$$
C_{1}=\frac{1-5 v_{s}^{2}}{3-5 v_{s}^{2}}, \quad C_{2} \sim \mathcal{O}\left(\mathcal{A}^{4}\right)
$$

Accordingly, it yields,

$$
v_{s} \simeq \frac{1}{\sqrt{5}}-\frac{2 \mathcal{A}}{\sqrt{5}}+\mathcal{O}\left(\mathcal{A}^{2}\right) .
$$

So the speed of the sound wave is shifted shown as (5.13). Then the equation of motion for $z_{H, 1}$ could be derived from (5.7) by inserting the solution for $z_{H, 0}$ (5.8) (5.13) (5.11) and (5.12) similarly. However the resultant equation from (5.7) is too complicated to be solved analytically. Hence we expand $z_{H, 1}$ in the small $\mathcal{A}$ case as,

$$
z_{H, 1}(x)=\mathcal{X}(x)+\mathcal{A} \mathcal{Y}(x)+\mathcal{O}\left(\mathcal{A}^{2}\right) .
$$

By the expansion (5.14), we obtain the decoupled equation for $\mathcal{X}(x)$ in leading order $\mathcal{O}\left(\mathcal{A}^{0}\right)$ is,

$$
0=\mathcal{X}^{\prime \prime}+\frac{\left(1-3 x^{2}\right)}{x\left(1+x^{2}\right)} \mathcal{X}^{\prime}+\frac{4}{1+x^{2}} \mathcal{X}+\frac{8-20 \Gamma}{\sqrt{5}\left(1+x^{2}\right)}
$$

and the equation for $\mathcal{Y}(x)$ in the next to the leading order $\mathcal{O}\left(\mathcal{A}^{1}\right)$ is, 


$$
\begin{aligned}
0= & \mathcal{Y}^{\prime \prime}+\frac{-2\left(-1+x^{2}\right)^{2} \mathcal{X}^{\prime}+\left(1-2 x^{2}-3 x^{4}\right) \mathcal{Y}^{\prime}}{x\left(1+x^{2}\right)^{2}}+\frac{4\left(-1+x^{2}\right)^{2} \mathcal{X}+4\left(1+x^{2}\right) \mathcal{Y}}{\left(1+x^{2}\right)^{2}} \\
& +\frac{4-4 x^{6}+x^{4}(-22+125 \Gamma)+x^{2}(-46+205 \Gamma)}{2 \sqrt{5} x^{2}\left(1+x^{2}\right)^{2}}
\end{aligned}
$$

The solution for (5.15) (5.16) could be found as

$$
\begin{aligned}
\mathcal{X}(x)= & \frac{1}{\sqrt{5}}(5 \Gamma-2)+D_{1}\left(-1+x^{2}\right)+D_{2}\left(-2-\ln x+x^{2} \ln x\right) \\
\mathcal{Y}(x)= & \left(-1+x^{2}\right) E_{1}+E_{2}\left(-2-\ln x+x^{2} \ln x\right)+\frac{1}{40}(2 \sqrt{5}-165 \sqrt{5} \Gamma \\
& \left.-160 D_{2}-160 D_{2} \ln x-32 \sqrt{5} \ln x-8 \sqrt{5} \ln ^{2} x+8 \sqrt{5} x^{2} \ln ^{2} x-40 D_{2} \ln ^{2} x+40 x^{2} D_{2} \ln ^{2} x\right)
\end{aligned}
$$

where $D_{1,2}, E_{1,2}$ are the integration constants. Since we have required that $z_{H, 1}$ must be regular at the horizon, it yields

$$
E_{2}=\frac{1}{\sqrt{5} \mathcal{A}}, D_{2}=-\frac{1}{\sqrt{5}} .
$$

Then imposing the boundary condition (5.4) to (5.14) with (5.17), we obtain

$$
\begin{aligned}
D_{1} & =-\mathcal{A} E_{1}, \\
\Gamma & \simeq \frac{2}{5}+\frac{4}{5} \mathcal{A}+\mathcal{O}\left(\mathcal{A}^{2}\right),
\end{aligned}
$$

which shows how the sound wave attenuation is also shifted by $\mathcal{A}$.

\section{Summary and discussion}

In this paper, first we have studied the 5d effective theory of our black D0-D4 system by the dimensional reduction (Kaluza-Klein reduction). It contains an additional vector field to the $5 \mathrm{~d}$ effective theory of the original D4-brane system as [12]. Then employing the similar technique as [12, 33, the physical fluctuations in the $5 \mathrm{~d}$ effective theory of our D0-D4 system has also been studied. Although the dual field theory of the black D0-D4 system is not completely clear (also for the original D4-brane system), we calculate the speed of sound and the sound wave attenuation in the hydrodynamic limit by using our $5 \mathrm{~d}$ effective theory. Particularly, we focus on the small $\mathcal{A}$ expansion in our calculations since the parameter $\mathcal{A}$ is related to the $\theta$ angle (the number density of D0-branes) which should be very small in QCD.

While our solution of the gauge invariant variable (5.8) (5.14) is quite different, it could be able to return to [12] if expanded by small $\mathcal{A}$. Accordingly, it is allowed to compare our result with [12. (5.13) and (5.19) show the speed of sound and the sound wave attenuation are all shifted by the presence of the D0-branes and they return to [12] consistently if setting $\mathcal{A}=0$ (i.e. no D0-branes). Due to (1.2), (5.13) and (5.19) could be interpreted as the modification from the $\theta$ angle or the chiral potential to the speed of sound and the sound wave attenuation. In hydrodynamics, the speed of sound depends on the mass of Fermions and 
Bosons, also the temperature 33 . But our holographic result suggests an additional $\theta$ dependence or chiral potential dependence if the topological term of QCD or Yang-Mills theory is considered. And as the leading order modification from the topological term (the $\theta$ angle), it shows the speed of sound decreases while the sound wave attenuation increase: 13 .

Besides, we need to keep in mind the simplification and the approximation used in our calculations. First we do not consider the fluctuations from the vector since this part is less relevant to the sound mode [13, 34, so we simply turn off this part. However it may lead to some observable effects such as CVSE in hydrodynamics. Thus a future study about it would be interesting and natural. Second, the solution for $\kappa$ (the combination of the gauge invariant variable with $Z_{w}, Z_{f}, Z_{\Phi}$ ) has been chosen as $\kappa=0$. This solution for $\kappa$ is a rough choice while it is consistent with its equation of motion (4.17) (also consistent with its boundary condition in [12]). Therefore a further improvement to take into account the solution of $\kappa$ is also needed although it might not change the results about the sound modes qualitatively.

\section{Acknowledgments}

We would like to thank Chao $\mathrm{Wu}$ and Yi Yang for valuable comments and discussions. We thank the Tsinghua University, Beijing, for its kind hospitality during the program "String 2016", where this work was finalized.

\section{Appendix}

In this appendix, we collect the equations of motion for the fluctuations in the $5 \mathrm{~d}$ effective theory. From (3.7) at the linearized level, the equations for the fluctuations are

$$
\begin{gathered}
g^{a b}\left[\partial_{a} \partial_{b} \delta f-\left(\Gamma_{a b}^{c} \partial_{c} \delta f+\Gamma_{a b}^{(1) c} \partial_{c} f\right)\right]-h^{a b} \nabla_{a} \nabla_{b} f-\frac{3}{80}\left(\frac{\partial^{2} \mathcal{P}}{\partial f \partial \Phi} \delta \Phi+\frac{\partial^{2} \mathcal{P}}{\partial f^{2}} \delta f+\frac{\partial^{2} \mathcal{P}}{\partial f \partial w} \delta w\right) \\
-\frac{1}{8} e^{\frac{10}{3} f+\frac{3}{2} \Phi}\left(2 F^{a b} \delta F_{a b}-h^{a c} g^{b d} F_{a b} F_{c d}-F_{a b} F_{c d} g^{a c} h^{b d}+\frac{10}{3} F_{a b} F^{a b} \delta f+\frac{3}{2} F_{a b} F^{a b} \delta \Phi\right)=0 \\
g^{a b}\left[\partial_{a} \partial_{b} \delta w-\left(\Gamma_{a b}^{c} \partial_{c} \delta w+\Gamma_{a b}^{(1) c} \partial_{c} w\right)\right]-h^{a b} \nabla_{a} \nabla_{b} w-\frac{1}{40}\left(\frac{\partial^{2} \mathcal{P}}{\partial w \partial \Phi} \delta \Phi+\frac{\partial^{2} \mathcal{P}}{\partial w^{2}} \delta w+\frac{\partial^{2} \mathcal{P}}{\partial w \partial f} \delta f\right)=0, \\
g^{a b}\left[\partial_{a} \partial_{b} \delta \Phi-\left(\Gamma_{a b}^{c} \partial_{c} \delta \Phi+\Gamma_{a b}^{(1) c} \partial_{c} \Phi\right)\right]-h^{a b} \nabla_{a} \nabla_{b} \Phi-\left(\frac{\partial^{2} \mathcal{P}}{\partial \Phi^{2}} \delta \Phi+\frac{\partial^{2} \mathcal{P}}{\partial \Phi \partial f} \delta f+\frac{\partial^{2} \mathcal{P}}{\partial \Phi \partial w} \delta w\right) \\
-\frac{3}{2} e^{\frac{10}{3} f+\frac{3}{2} \Phi}\left(2 F^{a b} \delta F_{a b}-h^{a c} g^{b d} F_{a b} F_{c d}-F_{a b} F_{c d} g^{a c} h^{b d}+\frac{10}{3} F_{a b} F^{a b} \delta f+\frac{3}{2} F_{a b} F^{a b} \delta \Phi\right)=0, \\
\partial_{a}\left[\sqrt{-g} e^{\frac{10}{3} f+\frac{3}{2} \Phi}\left(\frac{10}{3} F^{a b} \delta f+\frac{3}{2} F^{a b} \delta \Phi+g^{a c} g^{b d} \delta F_{c d}-F_{c d} h^{c a} g^{b d}-F_{c d} g^{c a} h^{b d}\right)\right]=0,
\end{gathered}
$$

\footnotetext{
${ }^{13}$ We have just noted a recent work [39] which studies the same holographic system after submitting the first version of this manuscript to arXiv. And our result is qualitatively similar as 39.
} 


$$
\begin{gathered}
\frac{40}{3}\left(\partial_{a} f \partial_{b} \delta f+\partial_{a} \delta f \partial_{b} f\right)+20\left(\partial_{a} w \partial_{b} \delta w+\partial_{a} \delta w \partial_{b} w\right)+\frac{1}{2}\left(\partial_{a} \Phi \partial_{b} \delta \Phi+\partial_{a} \delta \Phi \partial_{b} \Phi\right) \\
+\frac{1}{3} g_{a b}\left(\frac{\partial \mathcal{P}}{\partial f} \delta f+\frac{\partial \mathcal{P}}{\partial w} \delta w+\frac{\partial \mathcal{P}}{\partial \Phi} \delta \Phi\right)+\frac{1}{3} h_{a b} \mathcal{P}+\left(\frac{10}{3} \delta f+\frac{3}{2} \delta \Phi\right)\left(2 F_{c a} F_{b}^{c}-\frac{1}{3} g_{a b} F_{c d} F^{c d}\right) e^{\frac{10}{3} f+\frac{3}{2} \Phi} \\
+\left(2 \delta F_{c a} F_{b}^{c}+2 F_{a}^{c} \delta F_{c b}-2 F_{c a} F_{d b} h^{c d}-\frac{2}{3} g_{a b} F^{c d} \delta F_{c d}+\frac{2}{3} g_{a b} F_{c}^{d} F_{d e} h^{c e}-\frac{1}{3} h_{a b} F_{c d} F^{c d}\right) e^{\frac{10}{3} f+\frac{3}{2} \Phi}=\mathcal{R}_{a b}^{(1)} .
\end{gathered}
$$

where $\Gamma_{a b}^{c}$ and $\Gamma_{a b}^{(1) c}$ are defined as,

$$
\begin{aligned}
h^{a b} & =g^{a c} g^{b d} h_{c d}, \\
\Gamma_{a b}^{c} & =\frac{1}{2} g^{d c}\left(\partial_{b} g_{d a}+\partial_{a} g_{d b}-\partial_{d} g_{a b}\right), \\
\Gamma_{a b}^{(1) c} & =\frac{1}{2}\left[g^{c d}\left(\partial_{a} h_{d b}+\partial_{b} h_{a d}-\partial_{d} h_{a b}\right)-h^{c d}\left(\partial_{a} g_{d b}+\partial_{b} g_{a d}-\partial_{d} g_{a b}\right)\right] .
\end{aligned}
$$

and $\mathcal{R}_{a b}^{(1)}$ is defined as,

$$
\begin{aligned}
\mathcal{R}_{a b}^{(1)}= & \partial_{a} \Gamma_{c b}^{(1) c}-\partial_{b} \Gamma_{c a}^{(1) c}+\Gamma_{a d}^{(1) c} \Gamma_{c b}^{d}+\Gamma_{a d}^{c} \Gamma_{c b}^{(1) d} \\
& -\Gamma_{b d}^{(1) c} \Gamma_{c a}^{d}-\Gamma_{b d}^{c} \Gamma_{c a}^{(1) d} .
\end{aligned}
$$

The equations (4.5) - (4.14) could be obtained from (A1) - (A5).

\section{References}

[1] D.T.Son, A.O.Starinets, "Minkowski-space correlators in AdS/CFT correspondence: recipe and applications", JHEP 0209 (2002) 042, arXiv:hep-th/0205051.

[2] G.Policastro, D.T.Son, A.O.Starinets, "From AdS/CFT correspondence to hydrodynamics", JHEP 0209:043,2002, arXiv:hep-th/0205052.

[3] Dam T. Son, Piotr Surowka, "Hydrodynamics with Triangle Anomalies", PhysRevLett.103.191601, arXiv:0906.5044.

[4] P. Kovtun, D. T. Son, A. O. Starinets, "Viscosity in Strongly Interacting Quantum Field Theories from Black Hole Physics", Phys.Rev.Lett. 94 (2005) 111601, arXiv:hep-th/0405231.

[5] G. Policastro, D.T. Son, A. O. Starinets, "Shear viscosity of strongly coupled N=4 supersymmetric Yang-Mills plasma", Phys.Rev.Lett.87 (2001) 081601, arXiv:hep-th/0104066].

[6] D. Teaney, "Effect of shear viscosity on spectra, elliptic flow, and Hanbury Brown- Twiss radii," Phys. Rev. C 68, 034913 (2003).

[7] E. Shuryak, "Why does the quark gluon plasma at RHIC behave as a nearly ideal fluid?," Prog. Part. Nucl. Phys. 53, 273 (2004) arXiv:hep-ph/0312227. 
[8] D. Molnar and M. Gyulassy, "Saturation of elliptic flow at RHIC: Results from the covariant elastic parton cascade model MPC," Nucl. Phys. A 697, 495 (2002) [Erratum-ibid. A 703, 893 (2002)] arXiv:nucl-th/0104073.

[9] Edward Witten, "Anti-de Sitter Space, Thermal Phase Transition, And Confinement In Gauge Theories", Adv.Theor.Math.Phys.2:505-532,1998, arXiv:hep-th/9803131.

[10] T. Sakai and S. Sugimoto, "Low energy hadron physics in holographic QCD," Prog. Theor. Phys. 113, 843 (2005) arXiv:hep-th/0412141.

[11] T. Sakai and S. Sugimoto, "More on a holographic dual of QCD," Prog. Theor. Phys. 114, 1083 (2006) arXiv:hep-th/0507073.

[12] Paolo Benincasa, Alex Buchel, "Hydrodynamics of Sakai-Sugimoto model in the quenched approximation", Phys.Lett.B640:108-115,2006, arXiv:hep-th/0605076.

[13] Chao Wu, Yidian Chen, Mei Huang, "Fluid/gravity correspondence: A nonconformal realization in compactified D4 branes", Phys.Rev. D93 (2016) no.6, 066005, arXiv:1508.04038.

[14] Chao Wu, Yidian Chen, Mei Huang, "Fluid/gravity correspondence: Second order transport coefficients in compactified D4-branes", arXiv:1604.07765.

[15] J. M. Maldacena, "The large N limit of superconformal field theories and supergravity," Adv. Theor. Math. Phys. 2, 231 (1998) [Int. J. Theor. Phys. 38, 1113 (1999)] arXiv:hep-th/9711200.

[16] O. Aharony, S. S. Gubser, J. M. Maldacena, H. Ooguri and Y. Oz, "Large N Field Theories, String Theory and Gravity", Phys. Rept. 323, 183 (2000) arXiv:hep-th/9905111.

[17] Chao Wu, Zhiguang Xiao, Da Zhou, "Sakai-Sugimoto model in D0-D4 background", Phys.Rev. D88 (2013) no.2, 026016, arXiv:1304.2111].

[18] J. Liao, "Chiral magnetic effect in heavy ion collisions", arXiv:1601.00381.

[19] D. E. Kharzeev, J. Liao, S. A. Voloshin, and G.Wang, "Chiral magnetic effect in high-energy nuclear collisions: A status report", Prog. Part. Nucl. Phys. 88, 1 (2016).

[20] Si-wen Li, Tuo Jia, "Matrix model and Holographic Baryons in the D0-D4 background", Phys. Rev. D 92, 046007 (2015), arXiv:1506.00068.

[21] Si-wen Li, Tuo Jia, "Three-body force for baryons from the D0-D4/D8 matrix model", PhysRevD.93.065051, arXiv:1602.02259].

[22] Wenhe Cai, Chao Wu, Zhiguang Xiao, "Baryons in the Sakai-Sugimoto model in the D0-D4 background", PhysRevD.90.106001, arXiv:1410.5549.

[23] Francesco Bigazzi, Aldo L. Cotrone, Roberto Sisca, "Notes on Theta Dependence in Holographic YangMills", JHEP08(2015)090, arXiv:1506.03826.

[24] Francesco Bigazzi, Aldo L. Cotrone, "Holographic QCD with Dynamical Flavors", JHEP01(2015)104, arXiv:1410.2443.

[25] Dam T. Son, Piotr Surowka, "Hydrodynamics with Triangle Anomalies", PhysRevLett.103.191601, arXiv:0906.5044. 
[26] Gautam Mandal, Takeshi Morita, "Gregory-Laflamme as the confinement/deconfinement transition in holographic QCD", JHEP 1109 (2011) 073, arXiv:1107.4048].

[27] Gautam Mandal, Takeshi Morita, "What is the gravity dual of the confinement/deconfinement transition in holographic QCD?", J.Phys.Conf.Ser. 343 (2012) 012079, arXiv:1111.5190.

[28] Si-wen Li, Andreas Schmitt, Qun Wang, "From holography towards real-world nuclear matter", PhysRevD.92.026006, arXiv:1505.04886].

[29] Si-wen Li, Tuo Jia, "Dynamically flavored description of holographic QCD in the presence of a magnetic field", arXiv:1604.07197].

[30] A. Buchel and J. T. Liu, "Universality of the shear viscosity in supergravity," Phys. Rev. Lett. 93, 090602 (2004) arXiv:hep-th/0311175.

[31] A. Buchel, "On universality of stress-energy tensor correlation functions in supergravity," Phys. Lett. B 609, 392 (2005) arXiv:hep-th/0408095.

[32] P. Kovtun, D. T. Son and A. O. Starinets, "Holography and hydrodynamics: Diffusion on stretched horizons," JHEP 0310, 064 (2003) arXiv:hep-th/0309213.

[33] Paolo Benincasa, Alex Buchel, Andrei O. Starinets, "Sound waves in strongly coupled non-conformal gauge theory plasma", Nucl.Phys.B733:160-187,2006, arXiv:hep-th/0507026.

[34] Pavel K. Kovtun, Andrei O. Starinets, "Quasinormal modes and holography", Phys. Rev. D.72.086009, arXiv:hep-th/0506184.

[35] O. Aharony, A. Buchel and A. Yarom, "Holographic renormalization of cascading gauge theories," Phys. Rev. D 72, 066003 (2005) arXiv:hep-th/0506002.

[36] Alex Buchel, "Transport properties of cascading gauge theories", Phys.Rev.D72:106002,2005, hep-th/0509083.

[37] J. L. F. Barb’on, A. Pasquinucci, Phys. Lett. B 458 (1999) 288, hep-th/9904190.

[38] Kenji Suzuki, "D0-D4 system and QCD_\{3+1\}", Phys.Rev. D63 (2001) 084011, arXiv:hep-th/0001057.

[39] Chao Wu, Yidian Chen, Mei Huang, "The vorticity induced chiral separation effect from the compactified D4-branes with smeared D0-brane charge", arXiv:1608.04922. 